\title{
Computed tomographic findings in three Pinschers affected by incomplete ossification of the humeral condyle
}

\author{
Roberto Tamburro ${ }^{1}$, Ilaria Cerasoli ${ }^{1}$, Rosaria Savino $^{1}$, Rossella Terragni $^{2}$, \\ Ladislav Stehlík ${ }^{3}$, Massimo Vignoli ${ }^{1}$ \\ ${ }^{1}$ University of Teramo, Faculty of Veterinary Medicine, Teramo, Italy \\ ${ }^{2}$ Pet Care Veterinary Clinic, Bologna, Italy \\ ${ }^{3}$ University of Veterinary and Pharmaceutical Sciences Brno, Faculty of Veterinary Medicine, \\ Small Animal Clinic, Brno, Czech Republic
}

Received December 28, 2019

Accepted September 14, 2020

\begin{abstract}
Incomplete ossification of the humeral condyle is a cause of forelimb lameness in dogs. Affected dogs are predisposed to humeral condylar fractures. Incomplete ossification of the humeral condyle has been reported in breeds such as Spaniel, Rottweiler, Labrador and Pug. Diagnosis is made by a radiographic or computed tomographic study of the elbow, while treatment includes the placement of a bone screw across the humeral condyle. This paper discusses the computed tomography findings of incomplete ossification of the humeral condyle in three Pinschers.
\end{abstract}

Canine, diagnostic imaging, humeral intercondylar fissure, humeral condylar fracture

Humeral condyle fractures from severe traumatic events are very common in dogs (Marcellin-Little et al. 1994; Rovesti et al. 1998). They occur less frequently following minor trauma or during normal physical activity. In these cases, the predisposing factor seems to be a bone disorder known as incomplete ossification of the humeral condyle (IOHC), which affects some dog breeds, such as Spaniel, Labrador, Rottweiler and Pug (Moores and Moores 2017). According to some authors, IOHC is suspected to have a hereditary component, as it is linked to an autosomal recessive disorder (Marcellin-Little et al. 1994). Male subjects seem to be more predisposed than females, because oestrogen is believed to have a stimulating effect on epiphyseal fusion (Carrera et al. 2008). When we consider the pathogenesis of humeral condyle fracture, we must remember that condyle development is related to two separate ossification centres that appear around the $14^{\text {th }}$ postnatal day. The centres of lateral and medial ossification are separated during this period by a thin cartilaginous layer and their fusion takes place around the $70^{\text {th }}$ postnatal day (Hare 1961). Therefore, in skeletally mature animals the lateral and medial compartment of the humeral condyle should merge, with the cartilaginous layer undergoing complete ossification. In these subjects, fractures result from major trauma at the humeroradioulnar joint. An atraumatic fracture of the humeral condyle suggests instead an underlying abnormality along the condylar fusion zone. In dogs affected by IOHC, the cartilaginous layer that separates the lateral and medial portion of the humeral condyle does not undergo complete ossification; consequently, an area of fibrous tissue that separates the two portions of the condyle remains (Carrera et al. 2008). Diagnosis can be obtained by radiography of both elbow joints, since IOHC is often bilateral (Gnudi et al. 2005). Cranio-caudal oblique view with $15^{\circ}$ of supination provides a clear image of the humeral condyle (Rovesti et al. 1998). Frequently, however, radiographic evaluations yield disappointing results; that is why computed tomography images, in such cases, allow an excellent evaluation of the elbow joint, thus becoming the imaging technique of choice to diagnose IOHC (Carrera et al. 2008; Farrell et al. 2011).

Address for correspondence:

Ladislav Stehlík

Small Animal Clinic, Faculty of Veterinary Medicine

University of Veterinary and Pharmaceutical Sciences Brno

Palackého tř. 1946/1, 61242 Brno, Czech Republic 
This case report describes the computed tomographic findings in three cases of incomplete ossification of the humeral condyle in Pinschers, which, to the best of our knowledge, has never been published in the literature.

\section{Case details}

Three cases of IOHC-related fracture of the humeral condyle in Pinschers were included. Case 1 was a 2-year-old male weighing $3.5 \mathrm{~kg}$; Case 2 was a 2-year-old female weighing $3 \mathrm{~kg}$; and Case 3 was a 7-month-old male weighing $1.8 \mathrm{~kg}$. All patients presented lameness to the right thoracic limb with a sudden onset that did not result from any traumatic event. Clinical evaluation showed evidence of grade 4 lameness, pain on palpation and joint flexion/extension.

Dogs underwent the same work up including blood tests followed by bilateral radiographic evaluation and CT scan of both elbows performed under general anaesthesia. All patients were categorized as ASA (American Society of Anesthesiology classification) class II (a patient with mild systemic disease): they were premedicated with dexmedetomidine (Dexdomitor $0.5 \mathrm{mg} / \mathrm{ml}$, Zoetis, Italy) $(5 \mu \mathrm{g} / \mathrm{kg}$ ) and methadone (Semfortan $10 \mathrm{mg} / \mathrm{ml}$, Dechra, Italy) $(0.2 \mathrm{mg} / \mathrm{kg})$ intramuscularly (i.m.). Induction was achieved with propofol (Proposure $10 \mathrm{mg} / \mathrm{ml}$, Merial, Italy) to the effect intravenously. The trachea was intubated with an orotracheal tube (Magill) 4 ID and connected to a non-rebreathing system (modified T piece, Mapleson F) with a fresh gas flow of $500 \mathrm{ml} / \mathrm{kg} / \mathrm{min}$ with isoflurane in oxygen. Radiographs of the right elbow in cranio-caudal oblique view with $15^{\circ}$ supination were performed. Radiographic findings were consistent with a displaced fracture of the humeral condyle of the right elbow in all dogs (Plate V, Fig. 1). Suspecting IOHC in the absence of a known traumatic event, we proceeded to radiographic evaluation of the contralateral elbow; radiography results generated some doubts focusing on the presence of a radiolucent line between the lateral and medial portion of the humeral condyle (Plate V, Fig. 2). For this reason, CT scans were performed of both elbow joints in all three animals, to determine the extent of the injury, as well as to study more accurately any abnormalities of the left elbow. In all the cases a helical CT scanner (GE Pro-Speed Power, Milwaukee, WI, USA) was used. The examination was performed with animals in sternal recumbency with cranially extended thoracic limbs. Contiguous $1 \mathrm{~mm}$ thick slices of both elbow joints were collected. A high-frequency (bone) algorithm was used to reconstruct the images in the sagittal and dorsal plane. In all the examined cases, CT scans confirmed the complete fracture of the humeral condyle on the right side, without significant additional information compared to the X-ray images. The CT images confirmed also the presence of a vertical hypodense line between the lateral and medial portion of the left humeral condyle that extended from the joint surface proximally to the supratrochlear foramen (Plate VI, Figs 3, 4, Plate VII, Fig 5). On the basis of this information, we made a diagnosis of bilateral IOHC with displaced fracture of the humeral condyle of the right elbow.

\section{Discussion}

The IOHC is an emerging disease with an unknown pathogenesis (Moores et al. 2012; Moores et al. 2014; Moores and Moores 2017). Different grades of severity have been described: in some cases, there is no ossification while in others endochondral ossification leads to less resistant bone tissue that is therefore more prone to injury, including damages from physiological loads (Samoy et al. 2006). The IOHC was related to the asynchronous growth of the radius and ulna, a condition that leads to elbow dysplasia and a curved radius (Robin and Marcellin-Little 2001). This finding would justify the frequently observed coexistence of fragmentation of the medial coronoid process of the ulna and IOHC (Robin 
and Marcellin-Little 2001). Other authors found the presence of osteochondritis dissecans in two cases that were investigated for possible IOHC (Meyer-Lindenberg et al. 2002). However, we did not find either of these conditions in the animals we evaluated. To our knowledge, IOHC has been never diagnosed in a Pinscher dog. A paper published in 2017 reported the surgical treatment of 33 immature dogs affected by Salter-Harris type IV fracture of the lateral aspect of the humeral condyle (Cinti et al. 2017). As Pinscher was the most frequently affected breed within the study (14/33), the authors encouraged further evaluation to assess whether Pinscher dogs may also be affected by $\mathrm{IOHC}$, as they were unable to confirm it in their study (Cinti et al. 2017).

In the present report, we documented that the fracture of the humeral condyle was not a consequence of a major trauma, but instead associated with the incomplete ossification of the humeral condyle. Elbow radiographs easily detected the humeral condylar fracture on one side but could not provide a clear diagnosis on the contralateral limb. Computed tomographic evaluation enabled visualization of the hypo-attenuating line that crossed the humeral condyle associated with sclerosis near the hypo-attenuating area, thus achieving a diagnosis of IOHC on the contralateral elbow (Plates VI, VII, Figs 3, 4, 5). Radiographic cranio-caudal oblique view with $15^{\circ}$ supination yields images of the radiolucent line in the humeral condyle, but CT scans should always be considered in identifying and characterizing IOHC, as they clearly show the length and thickness of the intercondylar line (Farrell et al. 2011).

Dogs affected by IOHC may be totally asymptomatic and, in such cases, lesions are diagnosed only occasionally. In other cases, patients are presented for insidious, often episodic lameness, which tends to be exacerbated by physical activity, or even for a complete fracture of the humeral condyle as the result of a minor trauma, as in the cases detailed here.

An IOHC prophylactic treatment may be achieved by placement of a transcondylar screw (Moores and Moores 2017). In growing dogs affected by Salter-Harris fracture and/or in adult dogs affected by the humeral condyle fracture as a consequence of the IOHC, multiple Kirshner wires or transcondylar screws associated with an antirotational supracondylar bone fixation have been described in veterinary literature (Cinti et al. 2017; Moores and Moores 2017).

In the present study, all dogs were referred to our clinic for an advanced imaging evaluation and the surgical procedure was performed by the referring veterinary surgeon.

In conclusion, this paper suggests that Pinschers may be affected by incomplete ossification of the humeral condyle. Imaging evaluation of both elbow joints including radiographs and CT scans should be performed; prophylactic surgical treatment may prevent the fracture of the humerus.

\section{References}

Carrera I, Hammond GJ, Sullivan M 2008: Computed tomographic features of incomplete ossification of the canine humeral condyle. Vet Surg 37: 226-231

Cinti F, Pisani G, Vezzoni L, Peirone B, Vezzoni A 2017: Kirschner wire fixation of salter-harris type iv fracture of the lateral aspect of the humeral condyle in growing dogs. A retrospective study of 35 fractures. Vet Comp Orthopaed 30: 62-68

Farrell M, Trevail T, Marshall W, Yeadon R, Carmichael S 2011: Computed tomographic documentation of the natural progression of humeral intracondylar fissure in a Cocker Spaniel. Vet Surg 40: 966-971

Gnudi G, Martini FM, Zanichelli S, Volta A, Bertoni G, Del Bue M, Borghetti P 2005: Incomplete humeral condylar fracture in two English Pointer dogs. Vet Comp Orthopaed 18: 243-245

Hare WC 1961: The ages at which the centers of ossification appear roentgenographically in the limb bones of the dog. Am J Vet Res 22: 825-835

Marcellin-Little DJ, Deyoung DJ, Ferris KK, Berry CM 1994: incomplete ossification of the humeral condyle in Spaniels. Vet Surg 23: 475-487

Meyer-Lindenberg A, Heinen V, Fehr M, Nolte I 2002: Incomplete ossification of the humeral condyle as the cause of lameness in dogs. Vet Comp Orthopaed 15: 187-194 
Moores AP, Agthe P, Schaafsma IA 2012: Prevalence of incomplete ossification of the humeral condyle and other abnormalities of the elbow in English Springer Spaniels. Vet Comp Orthopaed 25: 211-216

Moores AP, Tivers MS, Grierson J 2014: Clinical assessment of a shaft screw for stabilization of the humeral condyle in dogs. Vet Comp Orthopaed 27: 179-185

Moores AP, Moores AL 2017: The natural history of humeral intracondylar fissure: an observational study of 30 dogs. J Small Anim Pract 58: 337-341

Robin D, Marcellin-Little DJ 2001: Incomplete ossification of the humeral condyle in two Labrador Retrievers. J Small Anim Pract 42: 231-234

Rovesti GL, Fluckiger M, Margini A, Marcellin-Little DJ 1998: Fragmented coronoid process and incomplete ossification of the humeral condyle in a Rottweiler. Vet Surg 27: 354-357

Samoy Y, Van Ryssen B, Gielen I, Walschot N, Van Bree H 2006: Review of the literature: elbow incongruity in the dog. Vet Comp Orthopaed 19: 1-8 
Plate V

Tamburro R. et al.: Computed ... pp. 269-272

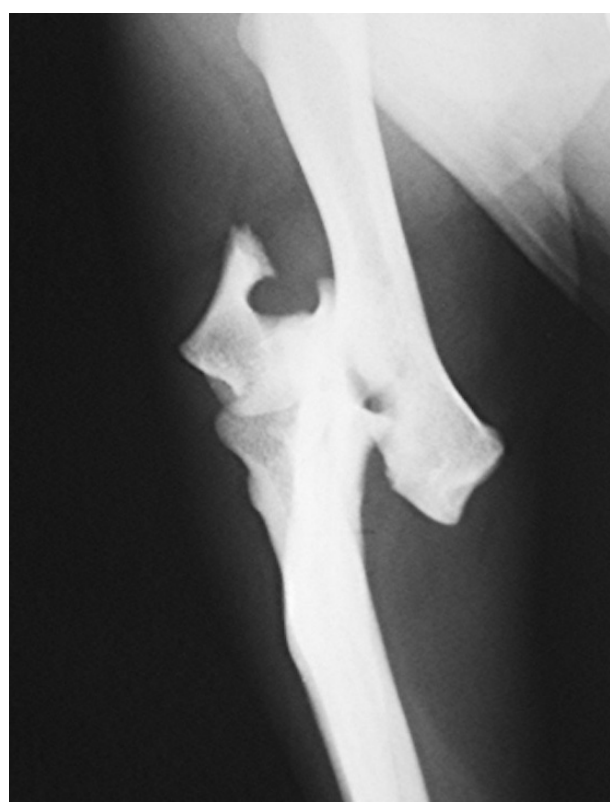

Fig. 1. Case 2: Craniocaudal view of the right elbow showing the displaced fracture of the humeral condyle with severe proximal displacement and overriding of the distal fragment.

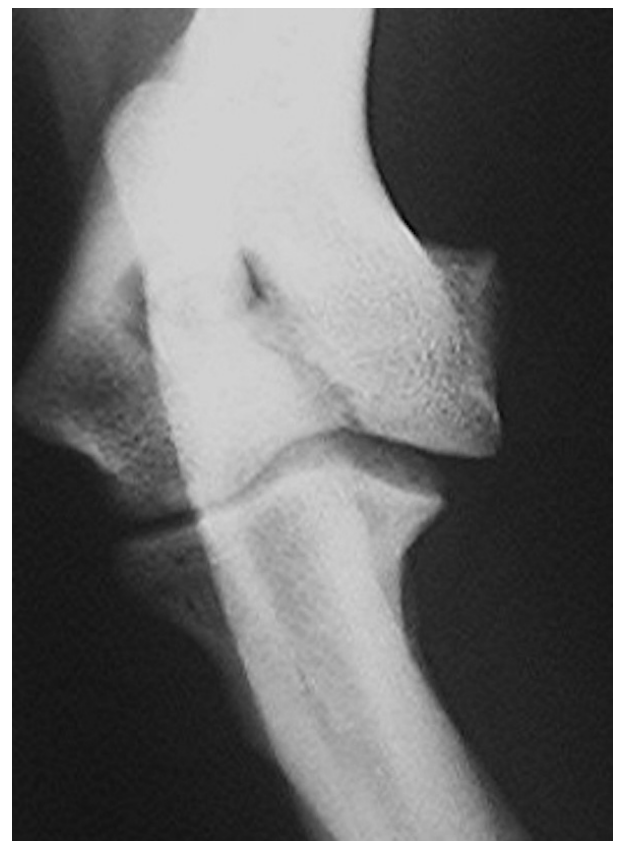

Fig. 2. Case 2: Cranio-caudal oblique view with $15^{\circ}$ supination of the left elbow. A radiolucent line is suspected at the humeral condyle. The partial overlap with the ulna and the possible Mach effect still leave some doubts about the final diagnosis. 


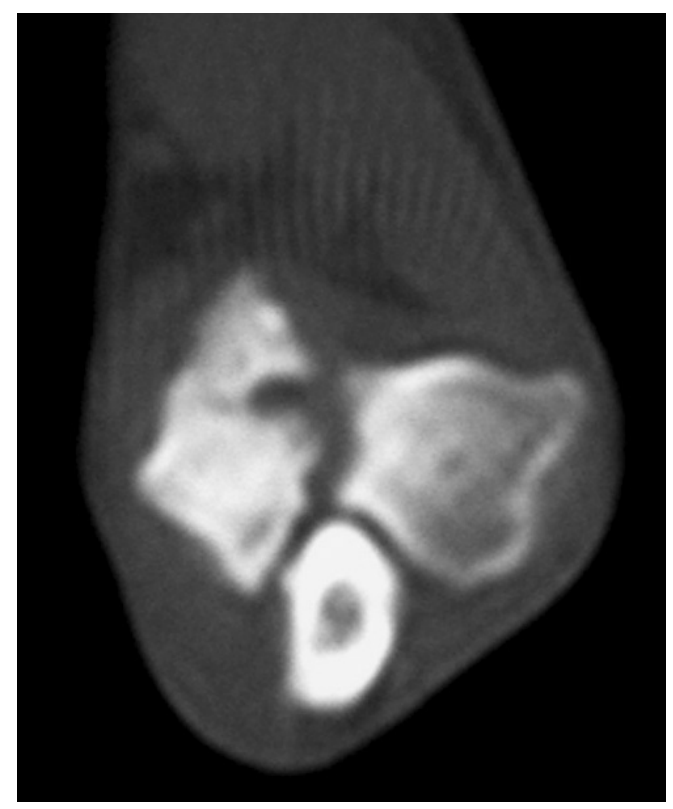

Fig. 3. Case 2: I ncomplete ossification of the humeral condyle (IOHC) is confirmed by the computed tomography (CT) scan in transversal plane. It is possible to see the hypo-attenuating line that crosses the humeral condyle and mild cranio-caudal dislocation of the medial and lateral parts of the condyle. Sclerosis at the edges of the hypo-attenuating area is well seen.

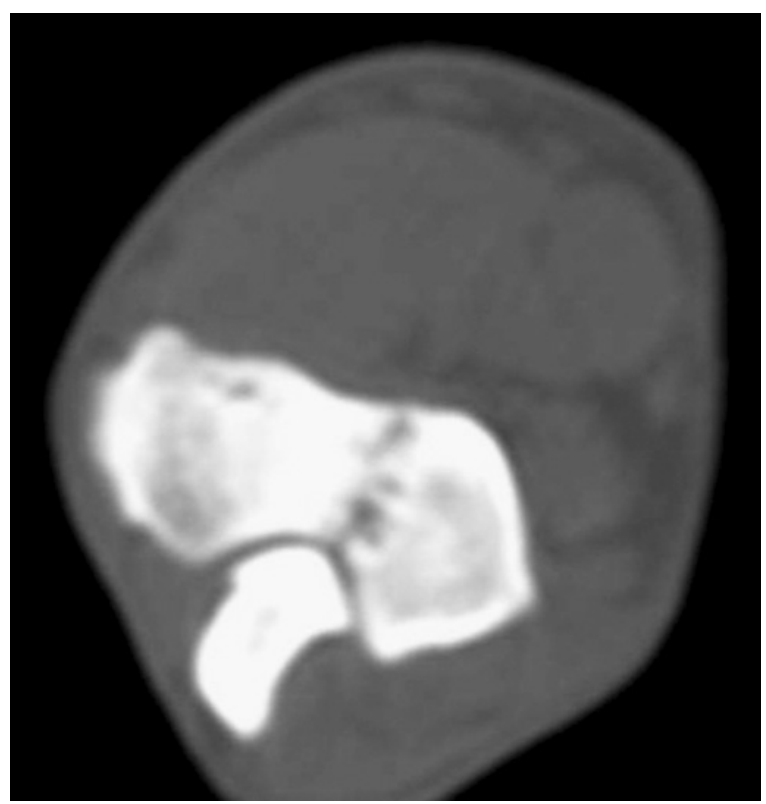

Fig. 4. Case 1: Left elbow computed tomography (CT) scan in the transversal plane. Hypodense line with mild sclerosis within the humeral condyle is seen. No dislocation of medial and lateral parts of the humeral condyle is visible. 


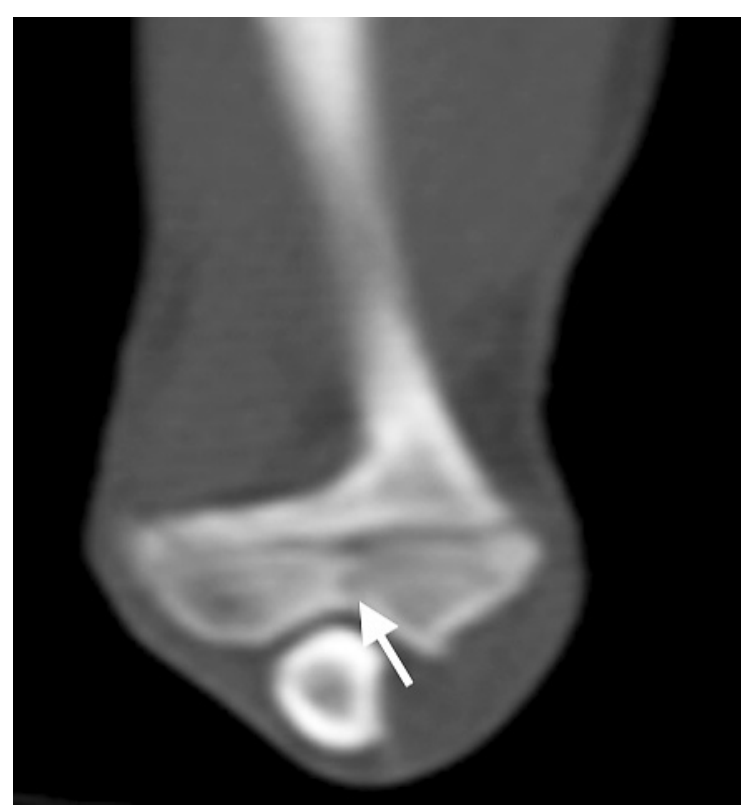

Fig. 5. Case 3: Left elbow computed tomography (CT) scan in the transversal plane. A hypodense line (white arrow) is visible within the humeral condyle. 